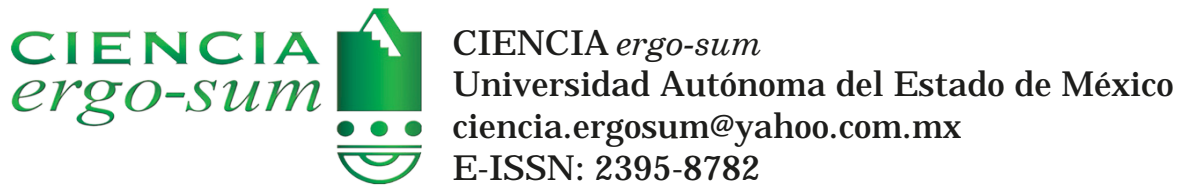

\title{
Probabilidades de detectar rayos gamma o cósmicos por HAWC con el juego del embaldosado libre
}

Tetlalmatzi Montiel, Margarita; Rodríguez Torres, Erika Elizabeth; Villarroel Flores, Rafael Probabilidades de detectar rayos gamma o cósmicos por HAWC con el juego del embaldosado libre

CIE NCIA ergo-sum, vol. 26, núm. 2, julio-octubre 2019|e55

Universidad Autónoma del Estado de México, México

Esta obra está bajo una Licencia Creative Commons Atribución-NoComercial-SinDerivar 4.0 Internacional.

Tetlalmatzi Montiel, M., Rodríguez Torres, E. E. y Villarroel Flores, R. (2019). Probabilidades de detectar rayos gamma o cósmicos por HAWC con el juego del embaldosado libre. CIENCIA ergo-sum, 26(2). https://doi. org/10.30878/ces.v26n2a9 


\title{
Probabilidades de detectar rayos gamma o cósmicos por HAWC con el juego del embaldosado libre
}

Probabilities of detecting gamma or cosmic rays by HAWC with the clean tile game

\author{
Margarita Tetlalmatzi Montiel \\ Universidad Autónoma del Estado de Hidalgo, México \\ tmontiel@uaeh.edu.mx \\ Erika Elizabeth Rodriguez Torres \\ Universidad Autónoma del Estado de Hidalgo, México \\ erikart@uaeh.edu.mx \\ Rafael Villarroel Flores \\ Universidad Autónoma del Estado de Hidalgo, México \\ rafaelv@uaeh.edu.mx
}

Recepción: 21 de febrero de 2018

Aprobación: 29 de agosto de 2018

\section{RESUMEN}

Se asocia el juego del embaldosado libre de Buffon con la probabilidad de que el observatorio HAWC (High Altitude Water Cherenkov) detecte cascadas creadas por rayos cósmicos y rayos gamma de altas energías. Para esto, se analizan todos los casos posibles del juego del embaldosado libre. Con la asociación realizada se tiene un modelo simple para calcular las probabilidades de que las cascadas caigan sobre HAWC y el anillo outriggers en tres casos posibles. Cada caso se estudia suponiendo la región ocupada por el observatorio como cuadrada o hexagonal. Se concluye que al considerar hexágonos las probabilidades son ligeramente mayores, pero podría ser más efectivo el considerar cuadrados. Los cálculos sólo requieren geometría básica.

Palabras clave: embaldosado libre, HaWC, outriggers.

\begin{abstract}
In this work the clean tile game of Buffon is associated with the probability that the observatory HAWC (High Altitude Water Cherenkov) detects showers created by cosmic rays and high energy gamma rays. For this, all possible cases of the clean tile game are analyzed. With the association, a simple model is provided to calculate the probabilities that the showers fall over HAWC and the ring of outriggers in three possible cases. Each case is studied assuming the region occupied by the observatory as square or hexagonal. It is concluded that, when considering hexagons, the probabilities are slightly higher, but it could be more effective to consider squares. The calculations only require basic geometry.
\end{abstract}

KEYWORDS: clean tile, HAWC, outriggers.

\section{INTRODUCCIÓN}

El observatorio de gran altura de agua Cherenkov (High Altitude Water Cherenkov, HAWC) recaba datos constantemente de los fenómenos más energéticos del universo. Con la información proporcionada se realiza un mapa celeste de rayos gamma de altas energías con objetos del universo antes no detectados (HAWC, 2017). En la actualidad se construye un anillo, llamado outriggers, alrededor de la zona principal de HAWC para aumentar su capacidad; por esta razón, es determinante analizar la forma y las capacidades de este anillo. Las herramientas usadas en estos análisis son en su mayoría muy sofisticadas (Capistrán et al., 2017). El enfoque que se presenta aquí puede dar una primera idea de estos análisis en una forma simple. 
Entre los rayos que llegan a la Tierra se encuentran los rayos cósmicos y los rayos gamma de altas energías del orden de $100 \mathrm{GeV}$ a $100 \mathrm{TeV}$. Estos rayos gamma pueden deber su origen a explosiones de supernovas o a la formación de estrellas (INAOE-HAWC, 2017). Cuando una partícula primaria de altísima energía llega a la Tierra e interactúa con la atmósfera produce una cascada de partículas secundarias que alcanzan velocidades mayores a la de la luz en la atmósfera. Al superarse la velocidad de la luz en algún medio, se emite una luz llamada radiación Cherenkov (Wikipedia, 2018).

El observatorio HAWK se encuentra a una altura de $4100 \mathrm{msnm}$, en la base del volcán Sierra Negra del Parque Nacional Pico de Orizaba, en Puebla, México. Consiste en un arreglo de 300 tanques principales que cubren un área de $22000 \mathrm{~m}^{2}$. El anillo outriggers, de 350 tanques pequeños, ocupará un área que es cuatro veces la de la zona de los tanques principales (Joshi y Jardin-Blicq 2017, HAWC, 2017). Cada tanque tiene en su interior detectores de radiación Cherenkov y están llenos de agua ultrapurificada y se encuentran totalmente a obscuras debido a que las partículas de las cascadas que entran a los tanques superan la velocidad de la luz en el agua y producen radiación Cherenkov dentro de ellos (Joshi y Jardin-Blicq, 2017). Cada uno de los tanques que registra la llegada de una cascada actúa como un pixel en la impresión o huella dejada por la cascada sobre el observatorio. Los rayos gamma de altas energías no son desviados por campos magnéticos; gracias a esto, al analizar los centros y las distribuciones de las cascadas, es posible discernir si éstas provienen de rayos cósmicos o rayos gamma de altas energías, así como conocer su procedencia y energía. Sin embargo, se tiene ambigüedad en la reconstrucción de los rayos gamma si los centros de las cascadas no quedan en las huellas, de aquí la importancia del anillo outriggers, pues, aunque son detectores secundarios, resultan efectivos para determinar estos centros (Capistrán et al., 2017; Joshi y Jardin-Blicq, 2017).

El juego del embaldosado libre fue planteado y resuelto por Georges Louis Leclerc conde de Buffon (Francia, 1707-1788) en un suplemento de su gran obra Histoire Naturelle (Buffon, 1777), donde dos jugadores lanzan una moneda a un piso con baldosas o mosaicos regulares y apuestan sobre si la moneda queda completamente dentro de un mosaico o si cubre de modo parcial más de uno. Los cálculos de las probabilidades de que se gane el juego se basan en ideas geométricas y marcan este problema, junto con otros, el inicio de la probabilidad geométrica (Mathai, 1999). Para relacionar la probabilidad de detectar las cascadas que caen sobre HAWC con el juego del embaldosado libre, se considera que se tiene un piso cubierto por mosaicos y que la región ocupada por HAWC (la zona principal junto con el outrigger) es uno de ellos. También se supone que las huellas dejadas por las cascadas de rayos secundarios en HAWC son circulares y así se asocia su registro con la caída de una moneda lanzada al azar sobre un piso embaldosado. Esto nos da un método muy simple para calcular la probabilidad de los lugares donde caen las huellas en la zona de HAWC.

Primero se aborda el problema del juego del embaldosado libre y se calculan las probabilidades de todos los posibles casos donde puede caer la moneda al ser lanzada a un piso con mosaicos regulares. Posteriormente, se calculan las probabilidades de que una huella que cae sobre la zona ocupada por HAWC y el outriggers descienda limpiamente, de que el centro de la cascada caiga en la zona de los detectores principales y de que un tercio del diámetro de la huella quede en la zona principal. En los cálculos se consideran mosaicos cuadrados y hexagonales con el objetivo de determinar la mejor forma para el outriggers. Todos los cálculos únicamente emplean geometría básica.

\section{El problema del embaldosado libre}

En el juego del embaldosado libre se lanza una moneda a un piso con mosaicos regulares. Un jugador apuesta a que la moneda caiga libremente dentro de un mosaico y el otro a que sea sobre más de uno. Gana el jugador que acierte la región donde quede la moneda.

Los mosaicos del embaldosado son regulares, por lo que son polígonos regulares acomodados de forma que no se sobreponen ni queda espacio entre ellos, esto es, todos los lados de los mosaicos miden lo mismo y todos sus 
ángulos interiores son iguales. Quedan bien acomodados cuando la suma de los ángulos interiores de los vértices de los mosaicos que coinciden en un punto es de $360^{\circ}$. Los ángulos interiores del triángulo equilátero son de $60^{\circ}$, los del cuadrado son de $90^{\circ}$ y los del hexágono son de $120^{\circ}$, por lo que se pueden acomodar seis, cuatro y tres mosaicos de ellos respectivamente (figura 1). No se pueden usar pentágonos, ya que sus ángulos interiores miden $108^{\circ}$; si se acomodan tres de ellos y coinciden por uno de sus vértices, los ángulos suman $324^{\circ}$ y falta espacio para completar los $360^{\circ}$; si se colocan cuatro, los ángulos suman $432^{\circ}$ y se sobreponen los pentágonos. El menor número de polígonos que se pueden acomodar coincidiendo por un vértice es de tres, que corresponde al caso de los hexágonos. Los ángulos interiores de polígonos con más de seis lados miden más de $120^{\circ}$; por este motivo, al colocar tres mosaicos regulares con más de seis lados, se sobreponen. Por lo anterior, sólo quedan tres casos, que están ilustrados en la figura 1.

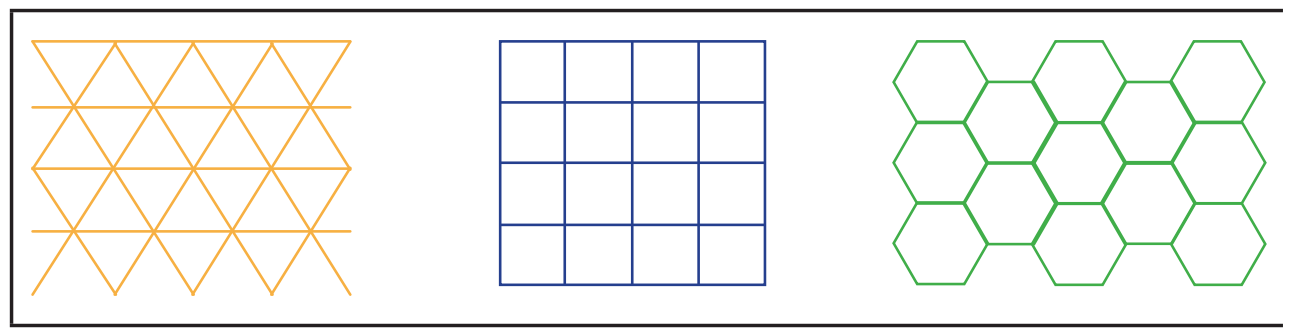

FIGURA 1

Embaldosados con mosaicos regulares

Fuente: elaboración propia.

En lo sucesivo, en el cálculo de las probabilidades se emplea la llamada probabilidad uniforme en el plano, esto es, si $S$ está en el plano y $A$ es un subconjunto adecuado de $S$ con área $|A|$, la probabilidad de que un punto caiga en $A$ es $P(A)=|A| /|S|$ (Hoel et al., 1971). Luego, si se representa un mosaico por $S$ y una cierta región de interés del mosaico por $A$, la probabilidad de que una moneda caiga en $A$ es el área de $A$ entre el área total del mosaico. Por las simetrías de los casos que ocurren en cada mosaico regular, es suficiente emplear solamente uno para el cálculo de las probabilidades. Dadas las dimensiones de HAWC y de la huella a considerar, se toma el lado del mosaico mayor al diámetro de la moneda.

A continuación se calculan las probabilidades de todos los casos donde puede quedar una moneda lanzada a un piso con embaldosado regular, ya que, hasta donde llega el conocimiento de los autores, sólo suele calcularse la probabilidad de que la moneda caiga libremente dentro de un mosaico o de que caiga sobre al menos dos.

\section{1. Embaldosado cuadrado}

En caso de tener un piso con mosaicos cuadrados, la moneda puede caer libremente en uno de ellos o entre dos, tres o cuatro (figura 2).

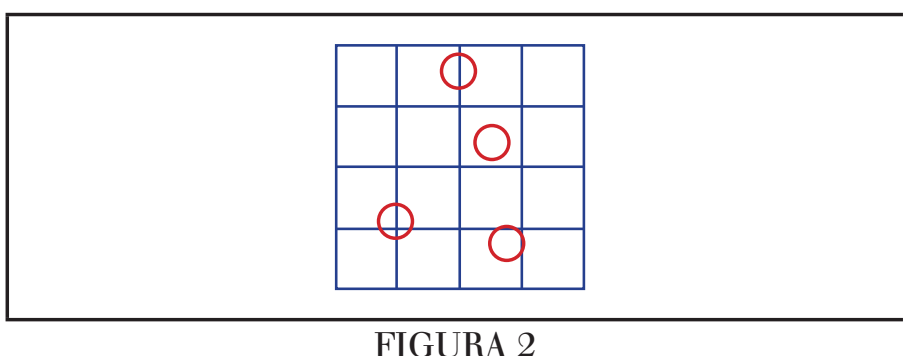

Monedas en mosaicos cuadrados

Fuente: elaboración propia. 
Considere un mosaico cuadrado de lado $L$ y una moneda con diámetro $d$, con el diámetro menor al lado. Para emplear la probabilidad uniforme, aquí $S$ corresponde a un mosaico cuadrado y los conjuntos $A$ son los conjuntos coloreados en verde, azul, naranja y amarillo de la figura 3. La moneda se representa por un círculo y su centro en rojo.

La moneda queda completamente dentro de un mosaico cuando su centro se encuentra en la región verde de la figura 3(a), que es un cuadrado de lado $L-d$. La probabilidad buscada se reduce, en este caso, al cociente de las áreas de un cuadrado de lado $L-d$ y otro de lado $L$, dando la probabilidad.

$$
P_{1}=\frac{(L-d)^{2}}{L^{2}}=\left(1-\frac{d}{L}\right)^{2}
$$

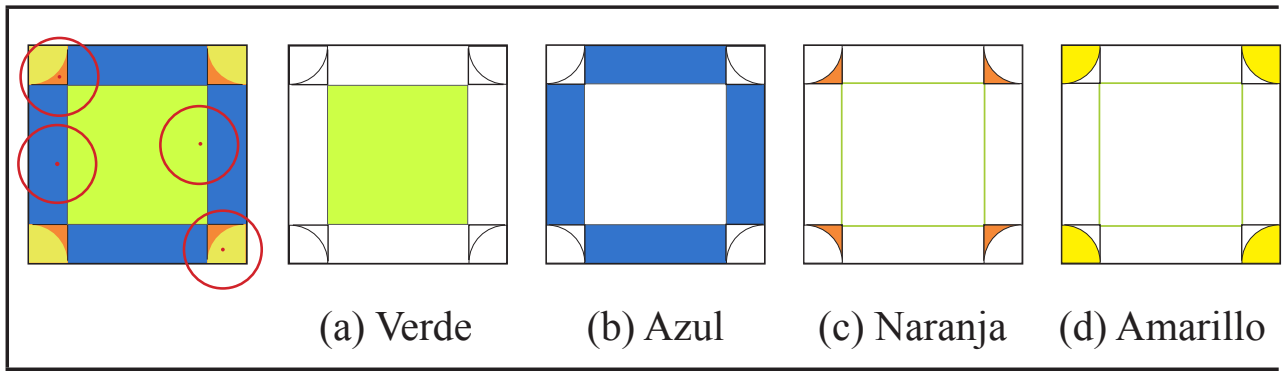

FIGURA 3

Mosaico cuadrado

Fuente: elaboración propia.

Nota: (a) La moneda toca un mosaico, (b) dos mosaicos, (c) tres mosaicos, (d) cuatro mosaicos.

Ahora, si la moneda queda entre dos cuadrados, su centro se encuentra en alguno de los cuatro rectángulos azules de la figura 3(b); estos son rectángulos de altura $d / 2$ y base $L-d$, quedando la probabilidad de este caso como $P_{2}=4\left(\frac{(L-d) d}{2}\right) / L^{2}=2\left(1-\frac{d}{L}\right) \frac{d}{L}$.

En caso que la moneda caiga sobre tres mosaicos, su centro debe estar en alguna de las secciones naranja de las cuatro esquinas de la figura 3(c). Juntando estas esquinas, la sección naranja es la diferencia de un cuadrado de lado $d$ y un círculo inscrito de diámetro $d$. Con ellos, la probabilidad buscada es $P_{3}=\frac{d^{2}-\pi d^{2} / 4}{L^{2}}=\frac{d^{2}}{L^{2}}\left(1-\frac{\pi}{4}\right)$.

Finalmente, el caso en el que la moneda corte cuatro bordes de mosaicos es precisamente cuando su centro queda en la región amarilla de la figura $3(\mathrm{~d})$, así $P_{4}=\left(\frac{\pi d^{2}}{4}\right) / L^{2}=\frac{\pi}{4} \frac{d^{2}}{L^{2}}$.

Pero ¿qué ocurre con el juego? Antes, es muy importante observar que la suma de todas las probabilidades es uno. Si el primer jugador apuesta a que la moneda quede completamente dentro de uno de los mosaicos y el otro a que quede sobre más de uno, los jugadores ganan con probabilidades $P_{1}$ y $P_{2}+P_{3}+P_{4}=1-P_{1}$ respectivamente.

\subsection{Embaldosado hexagonal}

Ahora, considere mosaicos hexagonales de lado $L$ y apotema $H$ y una moneda de diámetro $d$, con $L>d$. Ocurren tres casos: cuando la moneda queda dentro de un hexágono, cuando queda entre dos y tres (figura 4). Al emplear la probabilidad uniforme, aquí el conjunto $S$ es un mosaico hexagonal y los conjuntos $A$ son los conjuntos coloreados en verde, azul y naranja de la figura 4. Para calcular las áreas de estos conjuntos, básicamente se necesitan semejanzas de triángulos y la fórmula del área del hexágono. 


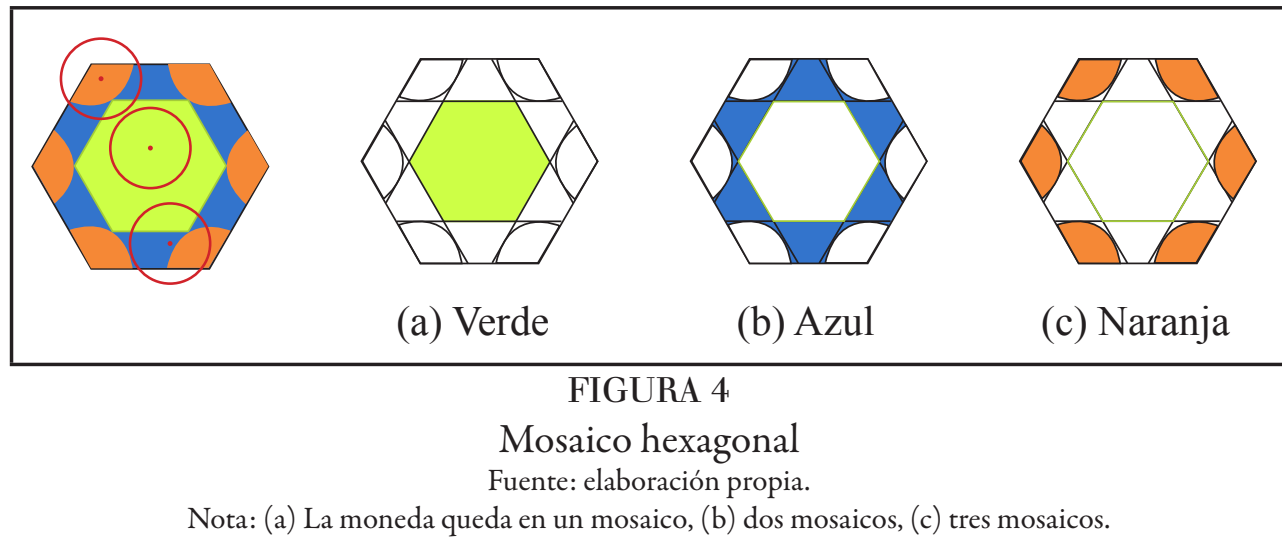

La moneda queda:

a) Libremente en el mosaico si su centro se encuentra en el hexágono verde de la figura 4(a) de lado $l=L-\frac{1}{\sqrt{3}} d$. La probabilidad de este caso es

$$
P_{1}=\frac{l^{2}}{L^{2}}=\frac{\left(L-\frac{d}{\sqrt{3}}\right)^{2}}{L^{2}}=\left(1-\frac{d}{\sqrt{3} L}\right)
$$

b) Entre dos hexágonos: cuando su centro queda en una de las seis secciones azul de la figura 4(b). Se puede encontrar que cada sección tiene área $\frac{d L}{2}-\frac{d^{2}}{6}\left(\sqrt{3}+\frac{\pi}{4}\right)$, con lo cual, la probabilidad es $P_{2}=\frac{3 d L-d^{2}\left(\sqrt{3}+\frac{\pi}{4}\right)}{\frac{3 \sqrt{3}}{2} L^{2}}=\frac{2 d}{3 \sqrt{3} L^{2}}\left(3 L-d\left(\sqrt{3}+\frac{\pi}{4}\right)\right)$.

c) Tocando exactamente tres hexágonos: el centro de la moneda queda en una de las seis secciones naranja de la figura $4(\mathrm{c})$; cada una tiene área $\frac{d^{2}}{12}\left(\sqrt{3}+\frac{\pi}{2}\right)$, y la probabilidad de que ocurra este caso es $P_{3}=\frac{\frac{d^{2}}{2}\left(\sqrt{3}+\frac{\pi}{2}\right)}{\frac{3 \sqrt{3}}{2} L^{2}}=\frac{d^{2}}{3 \sqrt{3} L^{2}}\left(\sqrt{3}+\frac{\pi}{2}\right)$.

\subsection{Embaldosado triangular}

En el caso de tener mosaicos regulares triangulares surgen muchas posibilidades, ya que la moneda puede cubrir parcialmente uno, dos, tres, cuatro o seis triángulos; estos casos dan las regiones verde, azul, naranja, púrpura y amarilla, respectivamente, de la figura 5. Los procedimientos para encontrar estas probabilidades son parecidos a los anteriores, por lo que sólo son indicados a continuación. En los cálculos se considera que $L>d$.

La probabilidad de que la moneda se encuentre:

a) Limpiamente en un triángulo, región verde delafigura $5(\mathrm{a})$, resultaser $P_{1}=\frac{\frac{\sqrt{3}}{4}(L-\sqrt{3} d)^{2}}{\frac{\sqrt{3}}{4} L^{2}}=\left(1-\frac{\sqrt{3} d}{L}\right)^{2}$. 


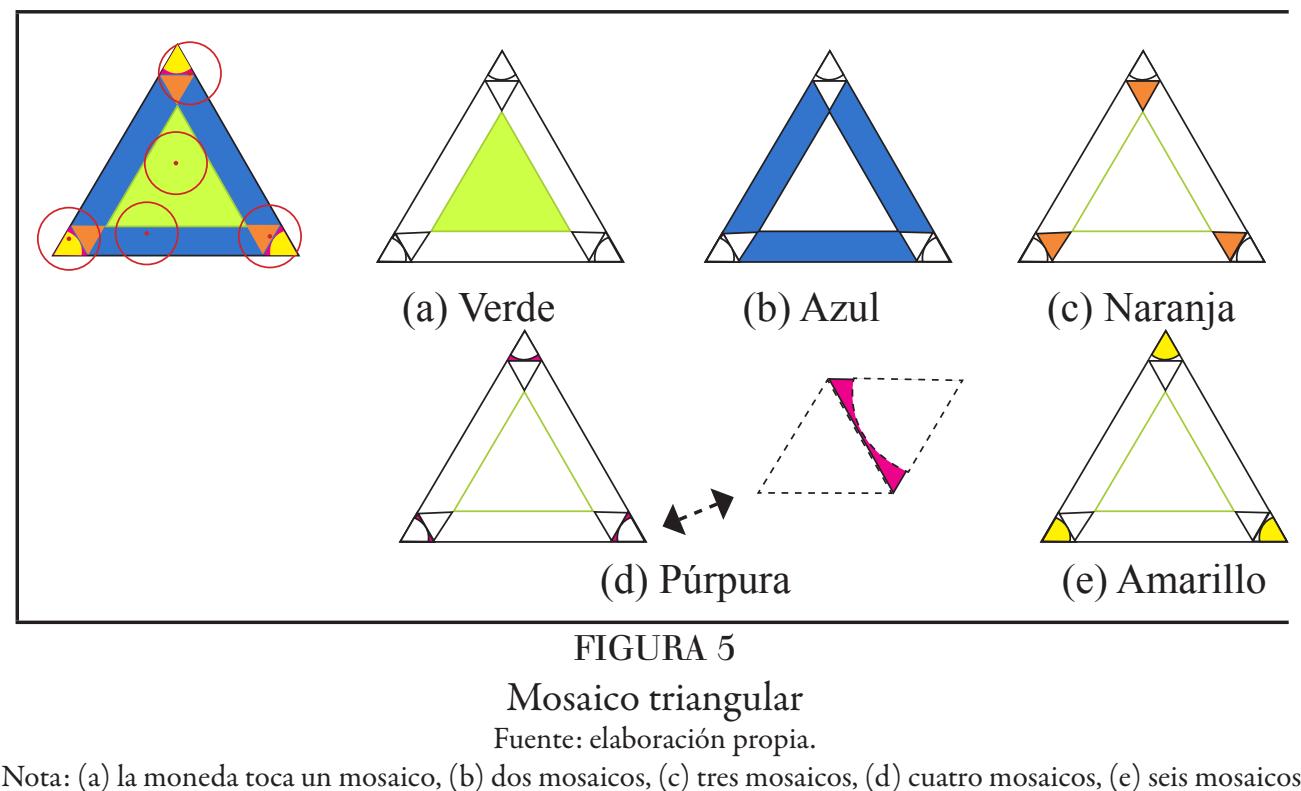

b) En exactamente dos triángulos, región azul de la figura 5(b), es

$P_{2}=\frac{\frac{3}{2} d\left(L-\frac{5 \sqrt{3}}{6} d\right)}{\frac{\sqrt{3}}{4} L^{2}}=\frac{2 \sqrt{3} d}{L^{2}}\left(L-\frac{5 \sqrt{3}}{6} d\right)$

c) Entre tres triángulos, región naranja de la figura 5(c), da $P_{3}=\frac{\frac{\sqrt{3}}{4} d^{2}}{\frac{\sqrt{3}}{4} L^{2}}=\frac{d^{2}}{L^{2}}$.

d) Sobre cuatro triángulos, región púrpura de la figura 5(d), es $P_{4}=\frac{d^{2} \frac{(\sqrt{3}-\pi / 2)}{4}}{\frac{\sqrt{3}}{4} L^{2}}=\frac{d^{2}}{\sqrt{3} L^{2}}\left(\sqrt{3}-\frac{\pi}{2}\right)$.

e) En seis triángulos, región amarilla de la figura 5(e), resulta con $P_{5}=\frac{\frac{\pi}{8} d^{2}}{\frac{\sqrt{3}}{4} L^{2}}=\frac{\pi d^{2}}{2 \sqrt{3} L^{2}}$.

Existen mucha páginas en internet relacionadas con el problema del embaldosado libre de Buffon, en particular se recomienda la página de Wolfram Math World (Weisstein, 2017), donde se pueden ver algunas animaciones.

\section{Aplicando a HAWC}

El área ocupada por el arreglo de los 300 tanques principales de HAWC es de aproximadamente $22000 \mathrm{~m}^{2}$. El área del anillo outriggers con 350 tanques secundarios será cuatro veces el área de la zona principal (Joshi y Jardin Blicq, 2017; HAWC, 2017). Cuando los centro de las cascadas quedan sobre HAWC, las huellas pueden tener más de $100 \mathrm{~m}$ de diámetro. Aquí se va a suponer que son circulares, de diámetro aproximado a $100 \mathrm{~m}$ y que caen en forma aleatoria sobre HAWC. 
Para relacionar la probabilidad de detectar las cascadas que caen sobre HAWC con el juego del embaldosado libre de Buffon, se considera: a) se tiene un piso cubierto por mosaicos de lado $L$, y la zona ocupada por HAWC junto con el outriggers es uno de estos mosaicos. b) En lugar de una huella circular se tiene una moneda de diámetro $d \cong 100 \mathrm{~m}$ lanzada al azar sobre el piso embaldosado. $c$ ) La zona de los detectores principales es un mosaico de lado $R$, centrado en el mosaico de lado $L$ y con un área aproximada de $22000 \mathrm{~m}^{2}$ (figura 6 y figura 8).

Es muy importante que para tener información relevante de una cascada una parte de su huella quede en la zona principal de HAWC. A continuación se calculan las probabilidades de tres casos: $a$ ) la huella queda limpiamente en la región de lado $L, b$ ) el centro de la huella queda en la región pequeña de lado $R$ y $c$ ) al menos un tercio del diámetro de la huella queda en la región pequeña. Primero cuando la región es cuadrada y luego cuando es hexagonal.

\section{1. Modelo cuadrado}

Considere dos cuadrados: uno pequeño de lado $R$ centrado en otro de lado $L$ (figura 6). Tomando el diámetro del círculo $d \cong 100 \mathrm{~m}$ y el cuadrado pequeño con área aproximada de $22000 \mathrm{~m}^{2}$, resulta $R \cong 148.3 \mathrm{~m}$. Como el área del anillo es cuatro veces el área donde están los detectores principales, es decir $L^{2}-R^{2} \cong 4 R^{2}$, entonces $L \cong \sqrt{5} R \cong 331.7 \mathrm{~m}$.

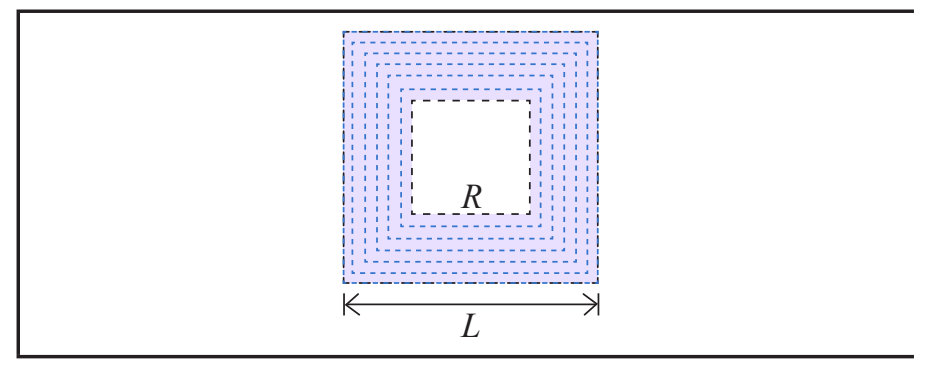

FIGURA 6

Detectores principales (blanco) y secundarios (azul)

Fuente: elaboración propia.

En la figura 7 se ilustran los tres casos señalados. En el caso 1 (figura $7($ a)) la huella cae limpiamente en el cuadrado de lado $L$; por la ecuación (1) la probabilidad de este caso es aproximadamente $(1-100 / 331.7)^{2} \cong 0.4879$. En los casos 2 y 3 las probabilidades son el cociente de la región sombreada correspondiente y el área del cuadrado de lado $L$. Para el caso 2 (figura $7(\mathrm{~b})$ ), por la relación $L^{2} \cong 5 R^{2}$ la probabilidad es aproximadamente $1 / 5$. Observando el caso 3 en la figura $7(\mathrm{c})$, la región sombreada es prácticamente el complemento del área de la figura 3(c), de un cuadrado de lado $R+d / 3$ y una moneda de diámetro $d / 3$, por lo que su área es $(R+d / 3)^{2}-\left((d / 3)^{2}-\pi(d / 6)^{2}\right)$, y la probabilidad de este caso es aproximadamente 0.2977 . Obsérvese que el caso 2 corresponde a cuando una moneda de diámetro $L-R$ queda limpiamente en un cuadrado de lado $L$. Ya que $\frac{1}{2}(L-R)=91.7$, si una huella toca el borde exterior del outriggers, resulta que $8.3 \mathrm{~m}$ de su diámetro queda en la zona principal.

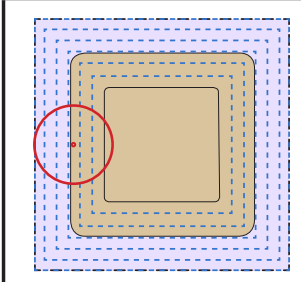

(a) Caso 1

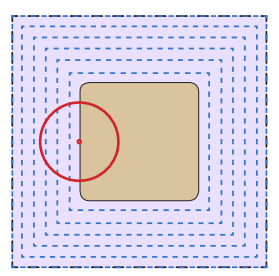

(b) Caso 2

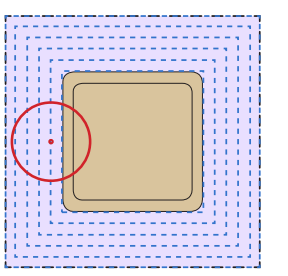

(c) Caso 3

FIGURA 7

Los tres casos con el modelo cuadrado

Fuente: elaboración propia. 


\section{2. Modelo hexagonal}

Ahora se tiene un hexágono pequeño de lado $R$ con área de $22000 \mathrm{~m}^{2}$ y apotema $h$, con lo cual $R \cong 92$ y $h \cong$ 79.7, centrado en un hexágono mayor de lado $L$ y apotema $H$ (figura 8 ). Se conserva que $L^{2}-R^{2} \cong 4 R^{2}$, así $L \cong \sqrt{5} R \cong 205.7$ y $H \cong 178.14$. Recuerde que $d \cong 100$.

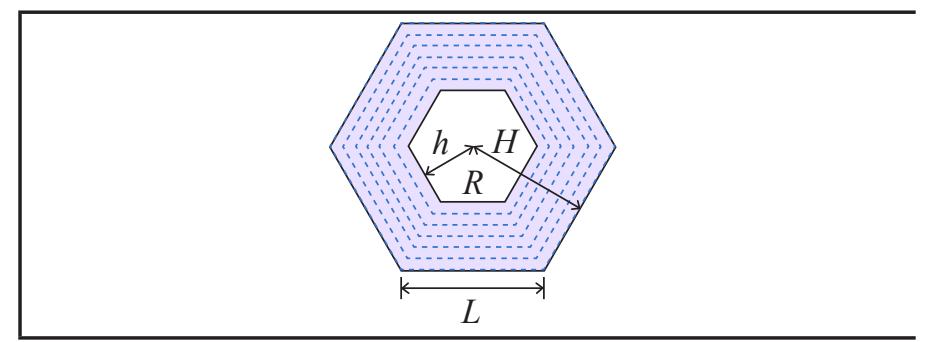

FIGURA 8

Detectores principales (blanco) y secundarios (azul)

Fuente: elaboración propia.

Los tres casos se ilustran con las regiones sombreadas de la figura 9. Los cálculos de los casos 1 y 2 (figuras 9(a) y 9(b)) son muy parecidos a los casos correspondientes del modelo cuadrado, sólo que ahora se emplea la ecuación (2). Para el caso 3 (figura 9(c)), como al menos un tercio del diámetro de la huella queda en el hexágono pequeño, la región es un hexágono de apotema $h+\frac{d}{6}$ al que se le resta la diferencia de un hexágono de apotema $\frac{d}{6}$ y un círculo de radio $\frac{d}{6}$ (las seis esquinas juntas), siendo esta probabilidad aproximadamente 0.2918 . Observe que el caso 2 corresponde a tener una moneda de radio $H-h$ al caer limpiamente en un mosaico hexagonal de lado $L$. Como $H-h \cong 98.44$, si una huella toca el borde exterior del outriggers, se tendrá que $1.56 \mathrm{~m}$ de su diámetro queda en la zona principal.

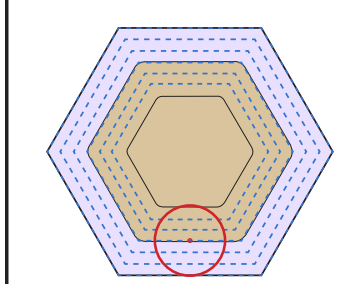

(a) Caso 1

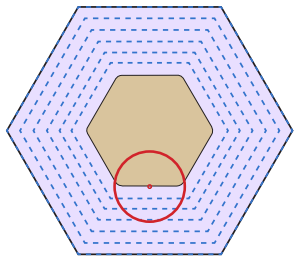

(b) Caso 2

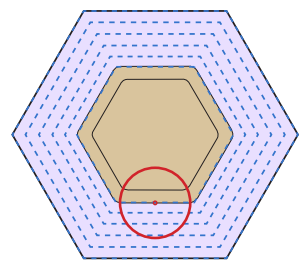

(c) Caso 3

FIGURA 9

Los tres casos con el modelo hexagonal

Fuente: elaboración propia.

Todos los cálculos anteriores se resumen en la tabla 1 .

TABLA 1

Probabilidades de acuerdo con los modelos cuadrado y hexagonal y cada caso

\begin{tabular}{lccc}
\hline Modelo & Caso 1 & Caso 2 & Caso 3 \\
\hline Cuadrado & 0.4879 & 0.2 & 0.2977 \\
Hexagonal & 0.5180 & 0.2 & 0.291 \\
\hline
\end{tabular}

Fuente: elaboración propia. 


\section{Conclusiones}

Los resultados muestran que la probabilidad de que la huella quede por completo dentro de la zona de los detectores principales junto con el anillo outriggers es mayor cuando se considera el modelo hexagonal, por lo que podría ser más eficiente un anillo outriggers hexagonal que uno cuadrado. Sin embargo, en el modelo cuadrado $8.3 \mathrm{~m}$ del diámetro de la huella queda en la zona principal y sólo $1.56 \mathrm{~m}$ en el modelo hexagonal aproximadamente, por lo que la información dejada por la huella puede ser más significativa con el modelo cuadrado. Por otro lado, la probabilidad de que al menos un tercio del diámetro de la huella quede dentro de los detectores principales es prácticamente la misma en ambos modelos.

\section{AnÁlisis Prospectivo}

El observatorio HAWC inició operaciones en 2015 y se espera que tenga una vida de al menos 10 años. Este observatorio es un sucesor del observatorio Milagro, al cual se le construyó un anillo outriggers que aumentó de manera drástica su capacidad, lo mismo se espera que ocurra con el anillo de HAWC (Joshi y Jardin Blicq, 2017). En el ámbito internacional hay un gran interés en el estudio de los rayos gamma de altas energías, por lo que es muy posible que se construyan en el futuro sucesores de HAWC.

Al relacionar el juego del embaldosado libre con la probabilidad de detectar las cascadas que caen sobre HAWC, se muestra un modelo muy simple para obtener resultados iniciales sobre la forma más conveniente de un anillo outriggers para un observatorio de tanques Cherenkov. Además, este modelo se puede emplear para ilustrar una aplicación de la probabilidad a la física en un salón de clase a nivel de licenciatura.

\section{Agradecimientos}

Agradecemos al Dr. Roberto Noriega Papaqui y al Dr. Pedro Miranda Romagnoli por sus valiosos comentarios y observaciones, así como a los árbitros por sus invaluables sugerencias.

\section{REFERENCIAS}

Buffon, G. (1777). Essai d'arithmétique morale. Histoire naturelle, générale et particulière, 4, 46-123.

Capistrán, T., Torres, I., \& Moreno, E. (2017). Characterization of the outer detector (outriggers) for HAWC. Journal of Physics: Conference Series, 792(012094). http://10.1088/1742-6596/792/1/012094.

HAWC (High Altitude Water Cherenkov). (2017). The high-altitude water Cherenkov gamma ray observatory. Disponible en https://www.hawc-observatory.org/. Consultado el 14 de junio de 2017.

Hoel, P. G., Port, S. C., \& Stone, C. J. (1971). Introduction to probability theory. Los Angeles: Houghton Mifflin Company.

INAOE-HAWC. (2017). High altitude water Cherenkov/ El observatorio de rayos gamma HAWC. Disponible en http://www.inaoep.mx/ hawc/. Consultado el 22 de junio de 2017

Joshi, V., \& Jardin-Blicq, A. (2017). HAWC high energy upgrade with a sparse outrigger array. Proceedings of Science. 35th International Cosmic Ray Conference-ICRC 2017. 
Mathai, A. M. (1999). An introduction to geometrical probability: distributional aspects with applications. Singapore: Gordon and Breach Science Publishers.

Weisstein, E. (2017). Clean tile problem. From Math World-A Wolfram Web Resource. Disponible en http://mathworld.wolfram.com/CleanTileProblem.html. Consultado el 14 de junio de 2017.

Wikipedia (13 de junio de 2018). Cherenkov radiation. Disponible en https://en.wikipedia.org/wiki/ Cherenkov_radiation. Consultado el 18 de junio de 2018.

\section{BY-NC-ND}

